\title{
Correction to: A bibliometric review on carbon accounting in social science during 1997-2020
}

\author{
Yikun Zheng ${ }^{1} \cdot$ Haishan $\mathrm{Yu}^{1} \cdot$ Yuquan Zhang $^{1}$ \\ Published online: 14 January 2022 \\ ๑) Springer-Verlag GmbH Germany, part of Springer Nature 2021
}

\section{Correction to: Environmental Science and Pollution Research https://doi.org/10.1007/s11356-021-17600-5}

1. The original HTML version of this Article contained a typographical error in the title of Figure 4 and Figure 5. "Fig.4 The academic collaborative relationships among the top 20 most productive countries" "Fig.5 The performance of the top 20 most productive countries" should read: "Fig.4 The academic collaborative relationships among the top 20 most productive countries/regions" "Fig. 5 The performance of the top 20 most productive countries/regions"

2. The original HTML version of this Article contained a typographical error in the "Results" section, under the subheading "The features of academic cooperation Publication features in different countries", where "The cooperation among the top 20 most productive countries is even, in which the USA plays a respective central role (Fig. 4)." should read: "The cooperation among the top 20 most productive countries/regions is even, in which the USA plays a respective central role (Fig. 4)."

The original article can be found online at https://doi.org/10.1007/ s11356-021-17600-5.

Haishan Yu

haishan.yu@sjtu.edu.cn

Yikun Zheng

mia.zheng.25@sjtu.edu.cn

Yuquan Zhang

yqwzhang@sjtu.edu.cn

1

\footnotetext{
China-UK Low Carbon College, Shanghai Jiao

Tong University, 3 Yinlian Rd Pudong New District,

Shanghai 201306, People's Republic of China
}

3. The original HTML version of this Article contained a typographical error in the "Methods and data" section, under the subheading "The bibliometric method", where "It was invented by Olle Persson in 1949." should read: "It was invented by Olle Persson in 1990s."

4. The original HTML version of this Article contained a typographical error in the "Research hotspots and frontiers" section, under the subheading "Keywords analysis", where "It was noted that the annual GHG emissions of China surpassed the USA in 2016." should read: "It was noted that the annual GHG emissions of China surpassed the USA in 2006."

Publisher's note Springer Nature remains neutral with regard to jurisdictional claims in published maps and institutional affiliations. 